\title{
Increasing the laser-acoustic method efficiency for testing metal products by using 3D visualization
}

\author{
I. R. Ismagilov ${ }^{1, *}, R$. I. Kalimullin ${ }^{1}$, and A. N. Borisov ${ }^{1}$ \\ ${ }^{1}$ Kazan State Power Engineering University, Kazan, Russia
}

\begin{abstract}
The article proposes a solution to the problem of increasing the efficiency of the laser-acoustic method of non-destructive testing. An approach to increasing the probability of detecting surface defects of microscopic dimensions in metals is considered. Due to the increased accuracy of determining defect parameters, the detail of $3 \mathrm{D}$ visualization of testing results was improved.
\end{abstract}

\section{Introduction}

The problems of non-destructive testing (NDT) of defects in metal products, which are the basis of various mechanical structures, have always been and remain relevant. The development of new methods of state-ofthe-art NDT and the strength resource of components, parts and products for responsible use is a key solution to the problem of improving the reliability of their operation and preventing emergency situations.

Acoustic method is one of the most reliable and constantly evolving methods of NDT [1-4]. It is based on the registration of oscillations generated or arising in an object. The acoustic method has several advantages compared with other methods of NDT such as high sensitivity, high performance and the ability to conduct a testing procedure directly at workplaces without interrupting the process.

Acoustic methods of NDT do not lose their relevance due to the fact that among them are developing methods that allow to excite acoustic oscillations in the control object (CO) without contact. Of particular interest are electromagnetic-acoustic (EMA) [1, 5-7] and laseracoustic methods. The last of them, based on the photoacoustic effect, is the most promising from the point of view of application in NDT devices [8-9]. There are works [10], describing the possibility of using a laser-acoustic method to detect defects in metal products obtained by both casting technologies and additive technologies, for example, by selective laser melting. Despite the fact that the testing method based on EMA transducers (EMAT) is considered to be non-contact, the signal-to-noise ratio and amplitude of the useful signal depend on the size of the gap between the object surface and EMAT [1]. Laser-acoustic technique is fully noncontact, as it allows to excite acoustic waves in control objects at distances greater than with EMAT. At the same time, contactless methods of detecting acoustic waves are mainly suitable for those cases when there are no additional noise and vibrations, that is, in laboratory conditions, and are hardly applicable in production.

The present work is a logical continuation of the researches conducted by the authors earlier [11-14] and aims to improve proposed laser-acoustic method in terms of the increase of defect detection sensitivity and probability of detection microcracks in metals. Also, it is important to get information about the shape of defect for determine the level of its criticality. The approach for $3 \mathrm{D}$ visualization of testing results proposed in this paper can increase reliability of NDT.

\section{Materials and methods}

In our earlier works $[11,12]$ an experimental setup and acoustic testing methods were presented, based on contactless ultrasound generation by pulsed laser radiation and acoustic wave registration by piezoelectric contact transducer. Proposed methods allow not only the detection of various types of defects in metals, but also their location and parameters (depth, width, length). In [13], for the first time, an attempt was made to develop a unified concept of laser-acoustic testing based on uniform equipment and analysis of surface acoustic wave (SAW) propagation parameters through a defect area when studying various control objects and defect types. Such an approach makes it possible to consider a combination of several methods as applied to different control objects as a single laser-acoustic method for detecting surface, near-surface and defects in metals and discontinuities in metal films.

Consider the main types of metal defects for which the development of the laser-acoustic method proposed in this article is aimed.

In order to select the best methods and control parameters, the defects are classified according to various criteria: according to the size of defects, according to their number and shape, according to the location of defects in the control object (CO), etc.

* Corresponding author: ildarism@yandex.ru 


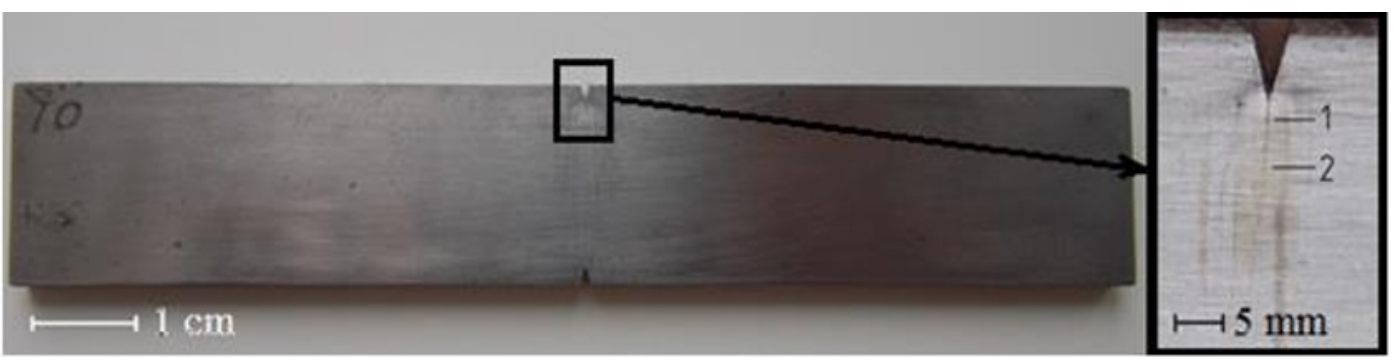

Fig. 1. Sample and an enlarged image of the crack formed in it after mechanical loading: 1 - scanning area No.1, 2-scanning area No.2.

When classifying defects by shape, we are interested in regular-shaped defects, oval, close to cylindrical or spherical, without sharp edges, as well as defects of an arbitrary, indefinite form, with sharp edges - cracks, tears, extraneous inclusions. When classifying defects by position, we will consider the following cases: surface defects located on the surface of a material, a semifinished product or a product are cracks, dents, foreign inclusions, as well as through defects.

The cross-sectional shape of through defects can be round (pores, fistula, slag inclusions) and slit-like (cracks, lack of penetration, structural defects, discontinuities at the locations of oxide and other inclusions and interlayers). By the nature of the internal surface, the through defects are divided into smooth and rough. The inner surface of the slag channels is relatively smooth. The internal surface of cracks, incomplete penetration and secondary pore channels, as a rule, is rough.

This list can be supplemented with defects in metallic materials synthesized using additive technologies, for example, selective laser melting. These include defects such as material discontinuity (cracks, pore aggregations) caused by crystallization stresses and shrinkage processes in the process of manufacturing parts [15].

In [14], we mentioned two modes of registration of Rayleigh wave. The first one is based on scanning the surface of an $\mathrm{CO}$ by a laser beam when a piezoelectric transducer (PET) fixedly mounted on the CO surface, the second one is based on the synchronous movement of the PET along with a laser beam with a fixed distance between them. The results of studies published in [1114] were obtained using only the first mode. The fixedly mounted PET has a more stable acoustic contact with the CO surface and can significantly reduce the instrumental error in analysing the amplitude of the detected surface wave in case of detection of discontinuities in metal films and near-surface defects in metal products. When monitoring surface defects, the first detection mode was used to implement a shadow method based on an analysis of the time it took for the surface wave to propagate from the generation region to the detection region with the rounding of defects. Registration only the time of passing the surface wave allows us to eliminate the problems associated with influence of the acoustic contact quality on signal-to-noise ratio. Thus, the first detection mode showed its high efficiency for detecting, localizing and sizing various types of defects.
But, in addition to this, in practice it is often necessary to determine the shape of a detectable defect, since this makes it possible to determine how dangerous it is from the point of view of structural failure. For example, defects of the correct shape, without sharp edges, are the least dangerous, since around them there is no concentration of mechanical stresses. Defects of a complex shape, for example, with sharp edges, are stress concentrators, the size of defects increases during the operation of the product along the lines of concentration of mechanical stresses, which, in turn, leads to its destruction.

\section{Main results}

It is possible to build the profile of surface defects based on the mathematical model described in [14]. Experimental data for imaging will be obtained by studying metallic samples with real surface cracks. For example, we conducted a study of the sample, which was a metal plate (Figure 1), in the middle of which a crack was formed as a result of mechanical loading.

The maximum crack width, as measured by the MicroScreener based on the LaboMet-I inverted metallographic microscope (version 2), at 40-fold magnification, was about 70 microns at the site of crack initiation (Figure 2). Further, the crack immediately narrowed to 40 microns, the field of which was observed a gradual decrease in its width. Scanning of this crack was made in area No. 1 (Figure 3) and area No. 2 (Figure 4), where the crack width was 30 and $10 \mu \mathrm{m}$, respectively.

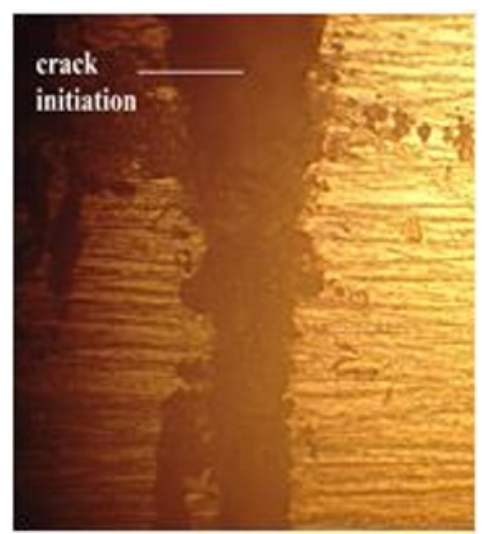

Fig. 2. Photograph of a crack obtained using a microscope in the area of crack initiation. 


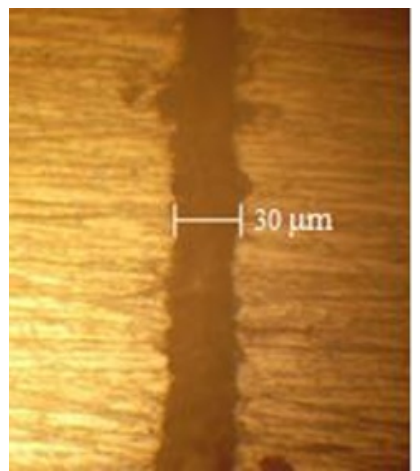

Fig. 3. Photograph of a crack obtained using a microscope in the area No. 1.

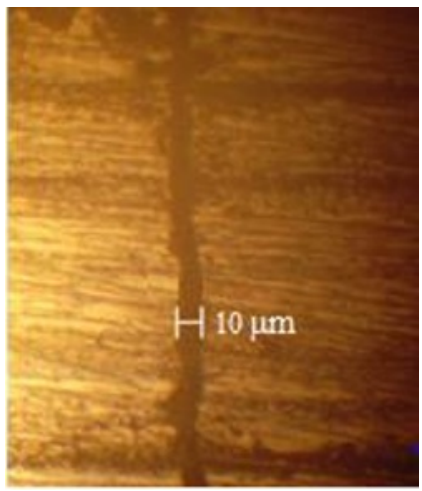

Fig. 4. Photograph of a crack obtained using a microscope in the area No. 2.

Having scanned the crack along its entire length with a step of $350 \mu \mathrm{m}$, we obtained a series of dependences $t$ $=f(L)$ and constructed the corresponding profiles for them $d=f(L)$. Consider the results of a crack scan in two areas (Figure 3,4). When the crack width was reduced, the algorithm for constructing a crack profile with respect to the time dependence turned out to be insensitive to such (small) defects, and we obtained a crack profile similar to the defect-free area, whereas the crack was observed in a microscope. Since the surface wave detection mode was used when the probe was stationary, the plot of $t=f(L)$ linearly increased with increasing distance between the generation and detection area L. cracks, which led to an increase in the time of passage of surface wave. At the same time, the graph shows that the linear increase was accompanied by small jumps in time values due to the quantization error even in the absence of a crack.

In order for the profile construction algorithm to be insensitive to such jumps and not to perceive them as a sign of the presence of a defect, we introduced a threshold value for the time change above which such jumps were perceived as discontinuity. This threshold value was chosen as the value of the time required by the surface wave to overcome the distance equal to the scan step at a certain surface wave propagation velocity (depending on the material of the control object). Such a solution, in our opinion, is the reason for the decrease in sensitivity to small inhomogeneities of the surface, which surface wave skirted in a time comparable to the threshold value. Reducing the threshold value is possible when using the surface wave registration mode, in which the laser beam and PET are moved simultaneously at a fixed distance from each other. This mode is convenient because in the time analysis of detected surface wave it is possible to monitor defects of a complex shape, since the change in the transit time of the surface wave occurs only if there are defects between the generation and detection areas. This is very important when implementing the process of reconstructing the crack profile in a specific control zone according to the dependence of the time of the surface wave passing from the generation area to the detection area, since in this case we can increase the sensitivity to detecting smaller defects and obtain their more detailed profiles for further visualization.
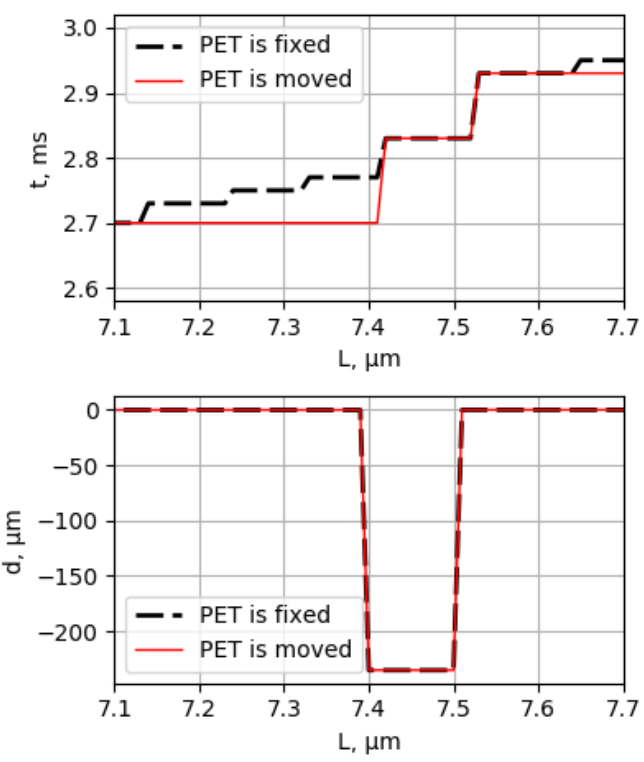

Fig. 5. Dependences $t=f(L)$ and $d=f(L)$ for area No. 1 of a microcrack with a fixed and movable PET.
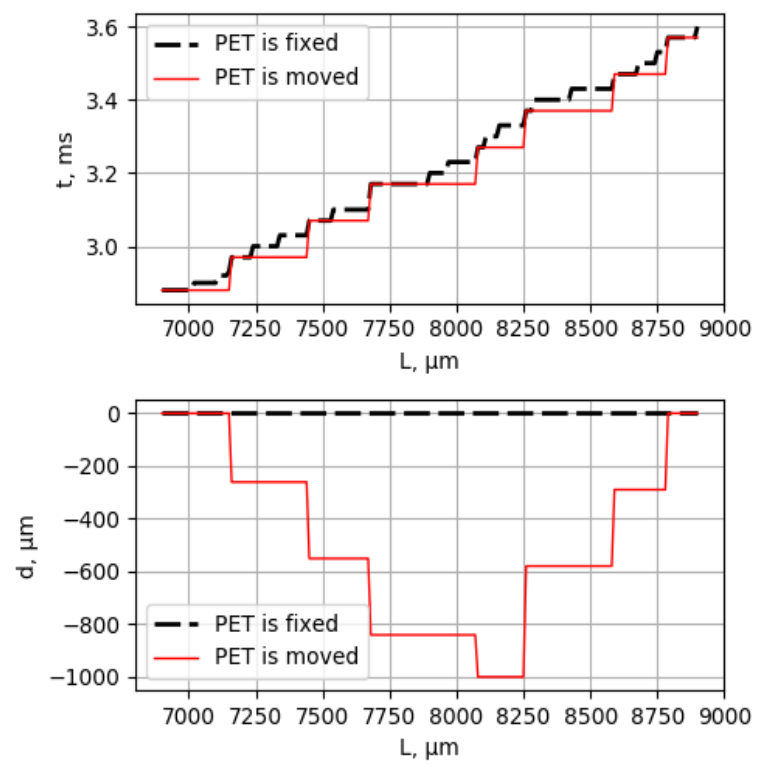

Fig. 6. Dependences $t=f(L)$ and $d=f(L)$ for area No. 2 of a microcrack with a fixed and movable PET. 
After scanning the areas shown in Figures 3, 4 in the mode of simultaneous displacement of the laser beam and PET, graphs were obtained $t=f(L)$, on the basis of which the profiles $d=f(L)$ were built (Figures 5 and 6 , red line). As can be seen from Figure 6, a change in the detection mode entailed the reconstruction of a more detailed profile of the scanned surface and made it possible to detect a microcrack, which had previously been missed.

Having collected together all the crack profiles obtained by discrete scanning along the crack length, we constructed its 3D image. Due to the fact that the length of the investigated crack is much greater than its depth and crack width, it is not possible to give an illustration of the full image in this article. Therefore, we present a fragment of the image in one of the scanning areas (Figure 7).

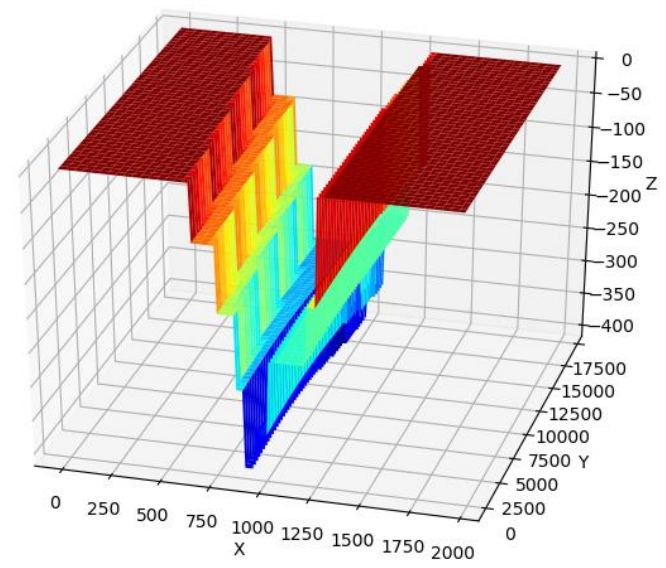

Fig. 7. A fragment of a three-dimensional image of a crack: X axis - crack profile, $\mu \mathrm{m}$; Y axis - crack length (crack fragment), $\mu \mathrm{m} ; \mathrm{Z}$ axis - crack depth, $\mu \mathrm{m}$.

\section{Conclusions}

Thus, by changing the mode of detection of surface waves, the possibilities of the previously proposed laseracoustic method in terms of detection of surface cracks with a width of less than 30 microns were expanded. The construction of three-dimensional images of defects on the basis of a number of profiles obtained by step-bystep scanning along the crack length, allows us to determine not only the geometrical parameters, but also the shape of the defect. In addition, improving the sensitivity of measurements contributes to obtaining more detailed visualization of surface defects necessary to assess their criticality. The efficiency of the method can be improved by reducing the quantization step by applying a continuous movement of the laser beamdetector system along the surface of the test object, for example, using a linear electric motor. In this situation, the scanning step will be determined by the ratio between the speed of movement and the repetition rate of laser pulses.

\section{References}

[1] I.N. Ermolov, Yu.V. Lange, Nondestructive Testing. Handbook in 7 Ultrasonic Testing, (Moscow: Mashinostroenie) 3, (2004)

[2] V.M. Ushakov, D.M. Davydov, L.I. Domozhirov, Detection and measurement of surface sracks by the ultrasonic method for evaluating fatigue failure of metals. Russian J. Nondestructive Testing 47(9), 631-41 (2011)

[3] V.A. Barkhatov, Development of Methods of Ultrasonic Nondestructive Testing of Welded Joints. Russian J. Nondestructive Testing 39(1), 23-47 (2003)

[4] A.B. Rinkevich, Yu.V. Korkh, Ya.G. Smorodinskii, Prospects of the Application of Nondestructive Testing to the Diagnostics of Nano- and Microstructural Materials. Russian J. Nondestructive Testing 46(1), 10-5 (2010)

[5] G.M. Suchkov, A.V. Donchenko, Real sensitivity of EMA instruments Russian J. Nondestructive Testing 43(6), 384-89 (2007)

[6] G.M. Suchkov, State-of-the-art capabilities of ema flaw detection. Russian J. Nondestructive Testing 41(12), 790-801 (2005)

[7] R.S. Edwards, S. Dixon, X. Jian, Depth gauging of defects using low frequency wideband Rayleigh waves Ultrasonics 44(1), pp 93-8 (2006)

[8] T. Windisch, Numerical and experimental studies towards improvements in laser-acoustic microscopy by optical-based sound beam shaping J. Phys.: Conf. Ser. 520, 1-7 (2014)

[9] S. Krishnaswamy, Theory and Application of Laser-Ultrasonic Techniques In Review of Progress in Quantitative Nondestructive Evaluation, D.O. Thompson and D.E. Chimenti, Eds. (New York: Plenum Press) 20A, 436-92 (2003)

[10] A.A. Popovich, D.V. Masaylo, V.S. Sufiiarov, E.V. Borisov, I.A. Polozov, V.A. Bychenok, I.Y. Kinzhagulov, I.V. Berkutov, D.S. Ashikhin, A.V. Il'inskii, A laser ultrasonic technique for studying the properties of products manufactured by additive technologies. Russian J. Nondestructive Testing 52(6), 303-9 (2016)

[11] V.A. Golenishchev-Kutuzov, I.R. Ismagilov, R.I. Kalimullin, S.A. Migachev, A.A. Khasanov, Subsurface crack dimensioning using the shadow method of laser-acoustic defectoscopy Power engineering: research, equipment, technology 5-6, 103-8 (2012)

[12] I.R. Ismagilov, R.I. Kalimullin, A.A. Khasanov, A.V. Moroz, S.A. Stepanov, Laser-acoustic testing of thin metal films Power engineering: research, equipment, technology 5-6, 24-7 (2013)

[13] I.R. Ismagilov, V.A. Golenishchev-Kutuzov, R.I. Kalimullin, S.A. Migachev, A.A. Khasanov, Detecting surface and volume defects in metals by the laser-acoustic method Russian J. Nondestructive Testing 50(6), 318-24 (2014)

[14] A.V. Golenishchev-Kutuzov, I.R. Ismagilov, R.I. Kalimullin, A.V. Semennikov, Through transmission technique of identification of surface cracks 
in metal items Mashinostroyeniye i inzhenernoye obrazovaniye 3, 32-7 (2014)

[15] N.P. Aleshin, M.V. Grigoriev, N.A. Shchipakov, V.V. Murashov, A.G. Evgenov, S.A. Vasilenko, I.S. Krasnov, The classification of flaws of metal materials synthesized by the selective laser melting method and the capabilities of nondestructive testing methods for their detection Russian $\mathrm{J}$. Nondestructive Testing. 52(1), 38-43 (2016) 